University of Nebraska - Lincoln

DigitalCommons@University of Nebraska - Lincoln

Faculty Publications in Computer \& Electronics Electrical \& Computer Engineering, Department Engineering (to 2015)

2008

\title{
ARTIFICIAL IMMUNE SYSTEM BASED IMAGE PATTERN RECOGNITION IN ENERGY EFFICIENT WIRELESS MULTIMEDIA SENSOR NETWORKS
}

\author{
Honggang Wang \\ University of Nebraska-Lincoln, hwang@unlnotes.unl.edu \\ Dongming Peng \\ University of Nebraska-Lincoln, dpeng2@unl.edu \\ Wei Wang \\ University of Nebraska-Lincoln, wwang@unlnotes.unl.edu \\ Hamid Sharif \\ University of Nebraska-Lincoln, hsharif@unl.edu \\ J. Wegiel \\ Raytheon Company, jerry_wegiel@raytheon.com \\ See next page for additional authors \\ Follow this and additional works at: https://digitalcommons.unl.edu/computerelectronicfacpub \\ Part of the Computer Engineering Commons
}

Wang, Honggang; Peng, Dongming; Wang, Wei; Sharif, Hamid; Wegiel, J.; Nguyen, D.; Bowne, R.; and Backhaus, C., "ARTIFICIAL IMMUNE SYSTEM BASED IMAGE PATTERN RECOGNITION IN ENERGY EFFICIENT WIRELESS MULTIMEDIA SENSOR NETWORKS" (2008). Faculty Publications in Computer \& Electronics Engineering (to 2015). 68.

https://digitalcommons.unl.edu/computerelectronicfacpub/68

This Article is brought to you for free and open access by the Electrical \& Computer Engineering, Department of at DigitalCommons@University of Nebraska - Lincoln. It has been accepted for inclusion in Faculty Publications in Computer \& Electronics Engineering (to 2015) by an authorized administrator of DigitalCommons@University of Nebraska - Lincoln. 


\section{Authors}

Honggang Wang, Dongming Peng, Wei Wang, Hamid Sharif, J. Wegiel, D. Nguyen, R. Bowne, and C. Backhaus 


\title{
ARTIFICIAL IMMUNE SYSTEM BASED IMAGE PATTERN RECOGNITION IN ENERGY EFFICIENT WIRELESS MULTIMEDIA SENSOR NETWORKS
}

\author{
H. Wang, D. Peng, W. Wang and H. Sharif, \{hwang, dpeng, wwang, hsharif\}@unlnotes.unl.edu \\ University of Nebraska-Lincoln, USA \\ J. Wegiel, D. Nguyen, R. Bowne and C. Backhaus, \{jerry_wegiel, dnguyen1, robert_w_bowne, \\ clint_p_backhaus\}@raytheon.com \\ Raytheon Company, USA
}

\begin{abstract}
In Wireless Multimedia Sensor Networks (WMSNs), low cost Complementary Metal Oxide Semiconductor (CMOS) camera sensors may only produce low resolution images due to hardware limitations. However, super-resolution images may be constructed from these low resolution images in a multiple sensor network, improving object pattern recognition success rates. There is a critical image recognition challenge in these reconstructed superresolution images for accuracy, complexity and limited energy resource in wireless sensor networks. Artificial Immune Systems (AIS), in particular those possessing algorithmic efficiency for image pattern differentiation, categorization and recognition, have potential advantages in low-cost automated monitoring and object detection applications. In this paper, we study the application of AIS for distributed and collaborative image pattern recognition in wireless multimedia sensor networks possessing energy efficient image communications and insitu image content processing. Our contributions are two fold. First, we propose an innovative approach involving dimension reduction to accelerate the AIS algorithm within an environment of low cost computing and efficient data transmission among the wireless sensor nodes. Second, a sleep control algorithm is proposed to reduce the image redundancies in order to achieve energy efficiency while guaranteeing the object recognition success rate in dynamic WMSN topology. Simulation results have demonstrated that the proposed approaches gain significant performance improvements in energy efficiency and in-network image content processing for WMSN. The algorithmic and simulation works are validated with the field data in collaborations between the University of Nebraska-Lincoln and Raytheon Company.
\end{abstract}

\section{INTRODUCTION}

In Wireless Multimedia Sensor Networks, image sensors provide in-network visual information for pattern recognition and classification, supporting many potential applications for military purposes as well as civilian applications such as agricultural environment monitoring or automatic surveillance. Many image-based pattern recognition methods have been proposed in recent years. However, most of them are involved with complex algorithms processing large size images and thus cannot be utilized directly in resource-limited sensor applications where either transmitting or processing power is limited and the size of images may be large which could consume high energy. Therefore, an energy-aware distributed image pattern recognition scheme is needed for WMSNs. In addition, the existing methods rarely consider hardware limitations in real sensor environments. For example, low cost CMOS image sensors often lack High Resolution (HR) since high precision optics and image sensors remain a cost concern in many commercial applications. A very promising approach is to use signal processing techniques to obtain an HR (or Super-Resolution) image from multiple observed low-resolution (LR) images [1]. The obtained HR image provides an opportunity for developing image pattern differentiation, categorization and recognition with high accuracy in WMSNs.

Large size image data processing for pattern discovery raises high computing overheads and energy consumption problem in WMSNs. With respect to this problem, Artificial Immune Systems (AIS) [2], possessing algorithmic efficiency for image pattern differentiation, categorization and recognition, have potential advantages. Biologically motivated artificial immune processing systems are able to recognize all cells (or molecules) within the body and categorize them in order to induce the defense mechanism. The important advantages of AIS algorithms include easy adaptation for data size reduction and more distinguishable data representation for pattern recognition. Since the image is large, it is difficult to recognize object patterns directly based on this raw data with high computation and communication costs. In combination with AIS, an effective solution is to perform dimensionality reduction and data re-representation for large size image data. The most common form of dimensionality reduction is Principal Component Analysis (PCA) [3]. Given a set of data, intuitively, PCA finds a linear lower-dimensional representation of this data set such that the variance of the reconstructed data is preserved. 
In this paper, we propose an innovative AIS-based image pattern recognition scheme in WMSNs. In this system, an AIS-based algorithm is designed for offline training of image recognition, which is conducted at the base station after the principal component of image is extracted [4]. We represent the principal component of each captured image as memory antibodies in AIS methods. The Euclidean distance among the principal components of the HR image that is reconstructed from the correlated LR images from each sensor are calculated as memory antibodies, and then are classified by an improved K-Nearest Neighbor (KNN) approach.

Further, the image transmission has to be performed with energy efficiency for image sensors to execute the object recognition. Thus, redundant visual information should be reduced in terms of both computational and transmission efficiencies in image sensor networks. In WMSNs, the total energy consumption and sensing coverage mainly depend on which sensors are active or asleep. With efficient sleep/wakeup scheduling, the redundant image frames could be reduced to save both communication and computation costs so long as image patter recognition can still be processed effectively. In this paper, a novel sleep scheme is proposed not only to achieve energy efficiency, but also to guarantee the performance of object recognition, which is different from many existing sleep control approaches.

The framework of our algorithm design is shown in Figure 1 , which includes two major parts - the image sensor node section and the base station part. The basic functionalities of image capturing, pre-processing, including PCA and Singular Value Decomposition (SVD) are performed at each image sensor node. At the base station, AIS and the KNN classification algorithm run for object recognition after each major component from the image sensor nodes is received and the HR principal components are reconstructed. The artificial immune network - based classification algorithm is designed to map the principal component of each HR image into a small amount of space so the feature can be easily discerned.

The rest of this paper is organized as follows. Section II provides a literature review on related works in WMSNs and AIS. Section III introduces the sleep/wakeup control mechanism being prepared for AIS and PCA-based image pattern recognition in WMSNs. Sections IV and V detail the image pattern recognition algorithms which are tailored to WMSNs with minimum data transmissions and computations among resource constrained image sensor nodes. Section VI demonstrates the experimental and simulation results, and the paper is concluded in Section VII.

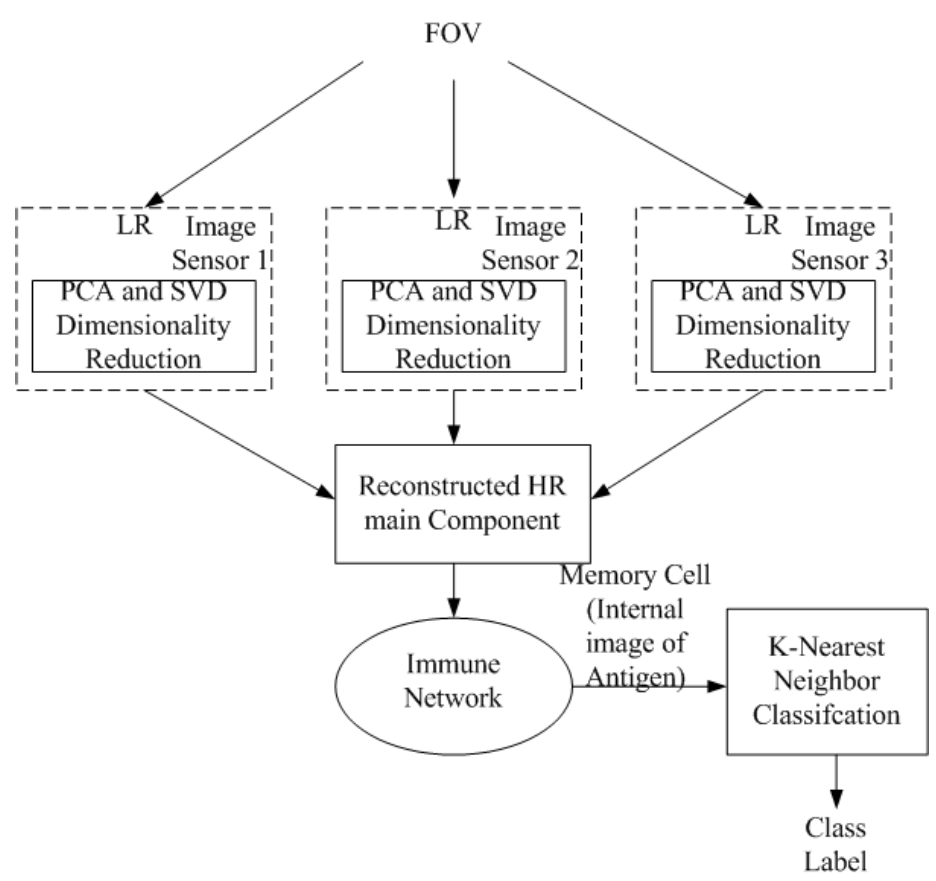

Figure 1. Distributed Image Pattern Classification
Based on Super-Resolution Using AIS Algorithm

\section{RELATED WORKS}

In articles [2, 5, 6], Leandro presented an artificial immune system architecture, which included three major algorithms: negative selection, clonal selection and immune networks. Unlike the clonal selection principle, the theory of immune network system provides an effective method to reduce the large data amount and is suitable for image pattern recognition. With the Immune Network (IN) algorithm for pattern recognition, the internal data representation can be more easily discerned and the data size can be significantly reduced through using Memory Cell representation. In [7], the authors presented a fast road classification and orientation estimation using omni-view images and neutral networks. A multisource classification model for remotely sensed data based on Markov Random Fields (MRF) is proposed in [8]. This approach is only suitable for multisource satellite imagery, which could not be generalized in WMSNs. A learning-based method to super-resolve face images using a kernel PCA-based prior model is presented in [9], which focuses on the specific application of face recognition. In [10], another kernel PCA has been suggested for various image processing tasks. A related image/video sensor coverage issue is also discussed in [11]. However, the above approaches have difficulties in applying the neural networks to the sensor-based object recognition due to their complexity and non energy-aware schemes for WMSNs. 
Some studies on sleep/wakeup control in sensor networks have been reported in literature [12]-[13]. In [12], the authors presented a power-aware methodology that uses an embedded micro operating system to reduce node energy consumption by exploiting both sleep state and active power management. A sleep scheduling algorithm in high density cluster-based sensor networks is proposed in [14]. Also, a wakeup scheme for sensor networks to balance energy saving and end-to-end delay is proposed in [13]. Although all these approaches could enhance energy efficiency in wireless sensor networks, they may not be energy efficient for image recognition in WMSNs where the image redundancy is one of major concerns. In this paper, the proposed wakeup/sleep scheme targets at the pattern recognition success performance with less energy costs, and the redundant image frames for pattern recognition are removed with efficient wakeup/sleep control.

\section{WAKEUP/SLEEP CONTROL FOR PCA/AIS IMAGE PATTERN RECOGNITION IN WMSNS}

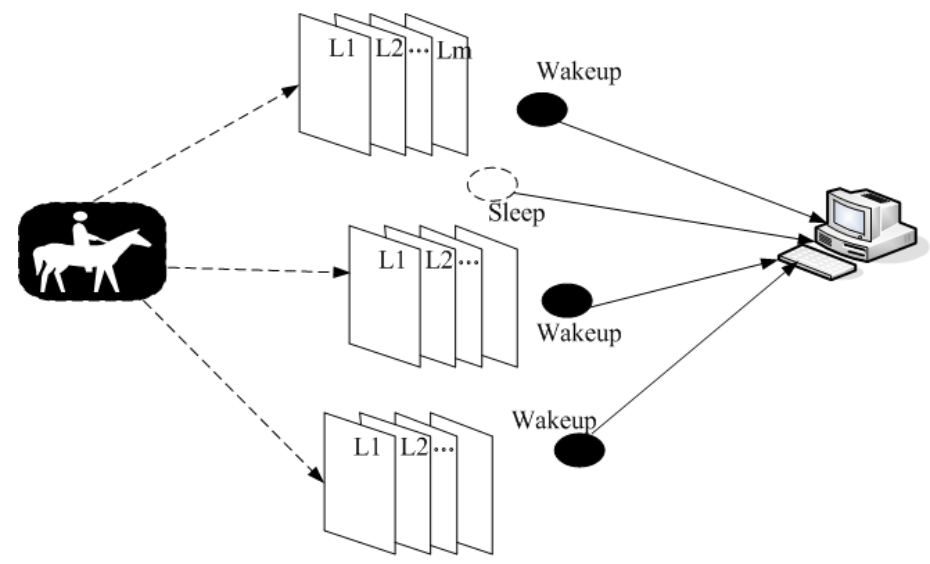

Figure 2. Sleep/Wakeup for image pattern recognition with energy efficiency

In WMSNs, the correlated sensors collaboratively observe the target, and the temporally changing images are continuously transmitted to the base station once the sensors are in a wakeup state. The pattern recognition performance depends on the number of image frames from sensors, which are used to construct the HR images. An extra number of image frames may not help the object recognitions once the recognition precision has been accomplished. The redundant image problem for pattern recognition can be solved by efficiently controlling sensor active and sleep. As shown in Figure 2, let vector $n=\left[n_{1}, n_{2}, \ldots n_{M}\right]$ denote the number of frames acquired from each sensor, i.e., $n_{i}$ frames from each sensor are acquired to deliver to cluster head or base station for pattern detection. $n_{i}$ is a positive integer, and
$\sum_{i=1}^{M} n_{i}=N$. The vector $\sigma=\left[\sigma_{1}, \ldots \sigma_{M}\right]$ is used to represent the characteristics of the sensors, with $\sigma_{i}$ defined as the probability of the $i_{t h}$ sensor being active. Let $\kappa(i)$ be the active/sleep indicator function on sensor $i$. $\kappa(i)=1$ indicates that sensor $i$ is active with probability $\sigma_{i}$. With probability $1-\sigma_{i} . \quad \kappa(i)=0$ denotes that the sensor is asleep.

$$
\Phi(n)=\operatorname{Pr} o b\left[\sum_{i=1}^{M} \kappa(i) \cdot n_{i} \geq T\right]
$$

$T$ is the least number of image frames that need to be acquired for pattern detection. $\Phi(n)$ is the pattern recognition success probability corresponding with threshold $T$.

The sensor's sleep/wakeup status depends on its residual energy within a cluster group in order to balance the lifetime among sensors. Therefore $\sigma_{i}$ can be expressed as the ratio between that specific sensor's residual energy and the total energy of sensors in the cluster.

$$
\sigma_{i}=\frac{E_{i}}{E_{\text {total }}}
$$

In this scheme, the target problem is to determine a threshold $T$ and the required probability of pattern recognition success rate, so that we could find optimal set of $\left[n_{1}^{*}, n_{2}^{*} \ldots n_{M}^{*}\right]$ image frames to be transmitted by each sensor based on their sleep/wakeup mechanism. This optimization problem is a k-out-of-n typical system problem, which can be solved with algorithms similar to the reliability evaluation of weighted-k-out-of-n systems in [15].

\section{PRINCIPAL COMPONENTS OF HR IMAGES FOR AIS}

As described in [9], each low-resolution image could be transformed by applying a geometric warp, blurring and down-sampling to the original super-resolution image with additional noise. There is a matrix-vector form expression as referred in [9]

$$
H_{i}=\Delta B_{i} G_{i} X+Z_{i}, \quad i=1,2, \ldots . r
$$

where $X$ is the HR image, $H_{i}$ is the $i t h$ LR image from the sensor, $Z_{i}$ is the noise in the $i t h$ observation, $\Delta$ is the decimation matrix while $B_{i}$ and $G_{i}$ are the blurring and geometric warp matrices, respectively for the ith observation. However, in terms of image pattern 
recognition applications, it is not necessary to transmit all the raw images to the base station if the pattern recognition can be done only based on the principal component of image. In our proposed algorithm, the principal component analysis (PCA) [3] is performed to extract the major components that are transmitted to the base station for pattern recognition. This is the further enchantment of the sleep/wake control algorithm in section III in order to reduce the images redundancies for pattern recognition. The proposed AIS-based algorithm can effectively recognize the pattern only based on the principal component of each image. There is a mathematical relation between SVD (Singular Value Decomposition) analysis [16] and PCA when principal components are calculated using the covariance matrix. The property of SVD is to provide the closest rank- $l$ approximation for a matrix $Y$, which can be used in image processing for compression and noise reduction. The difference between the proposed approach and the approach in [9] is that we only estimate the principal components of HR images instead of whole HR images. Hence, this approach only needs principal components of LR images from each sensor to be transmitted to the base station. The principal component of HR image could be reconstructed from these received LR principal components. Typical PCA algorithm can be referred in [7].

\section{AIS IMAGE RECOGNITION SCHEME}

The offline learning is an essential step to conduct the pattern recognition. Sub-section A describes the proposed method in details based on the study on AiNet [17].

\section{$A$. Offline training for pattern recognition at the base station}

At this phase, the trained images are selected and then, the PCA dimension reduction algorithm is applied to find the principal component of the image. The improved immune networks algorithm shown in Figure 3 is utilized to represent the image data in more distinguishable memory cell form. These memory cells of images are marked as the determined class types. In Figure 3, the Ag (Antigen)- Ab (Antibody) affinity is defined as the Euclidian distance. At the Clone \& Hypermutation block, the $n$ selected antibodies are going to proliferate (clone) proportionally to their antigenic affinity, generating a set $\mathrm{C}$ of clones. Record the colone classifcation type at the last colunm of C. At the Network pruning (Natural Death) and network suppresion pahse, eliminate those memory clones whose affinity is less than the pruning threshold. Finallly, it concatenates the total antibody memory matrix with the resultant clonal memory, and outputs image memory cells.

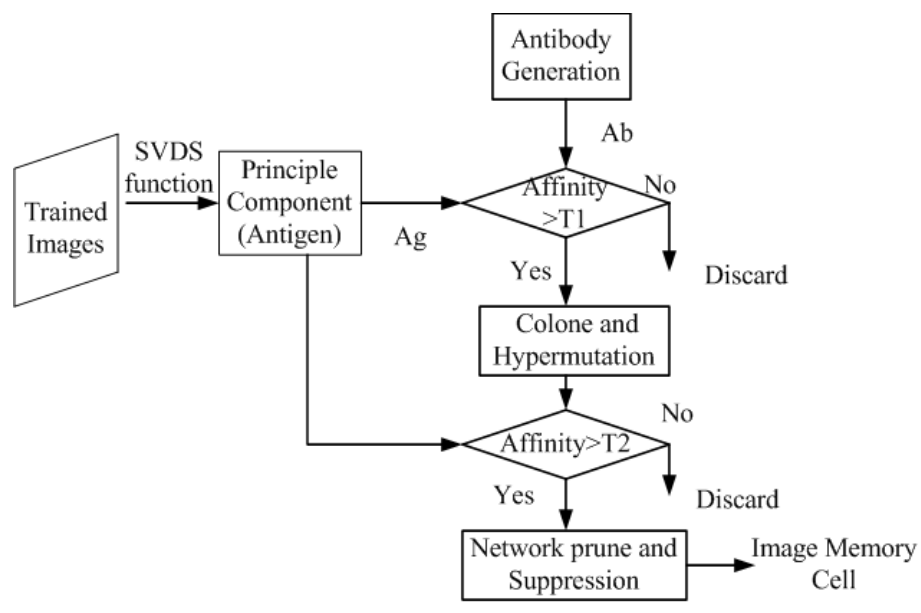

Figure 3. Image memory cell representation using artificial immune network

As described in [17], if the immune network has output $m$ memory cells, the computation time of the algorithm above required to compare all the elements of a matrix of size $m$ is $O\left(m^{\wedge} 2\right)$.

\section{B. Image capturing, processing and transmissions with sleep/wakeup control for AIS pattern recongion}

The detailed procedure of AIS-based algorithm is described as follows once the offline training has been completed.

Step 1: Each sensor of the same scene independently wakeup/sleep based on its residual energy.

Step 2: Each sensor captures images at its duty cycle period. The captured images are pre-processed. The principal components are extracted by the PCA method.

Step 3: The base station optimizes the number of frames with the determined pattern recognition success probability based on Equation (1). Then it informs each sensor the number of frames needed to be extracted with principal components and to be transmitted.

Step 4: The principal components are transmitted to the base station from sensors. The base station reconstructs the major component of HR resolution image. The improved KNN algorithm is used to classify the object based on trained and classified memory matrix from offline training.

Step 5: The base station outputs the classification results, and the object is recognized and classified.

\section{EXPERIMENTS AND SIMULATIONS}

We have designed an AIS-based image recognition algorithm to evaluate the proposed AIS algorithm, which 
provides processing, recognition and display as shown in Figure 3. This AIS algorithm has two parts. The training part is responsible for the training processing based on artificial immune network. The recognition part of the AIS algorithm handles the final classification and recognition process. Also, most parameters of the immune network training process can be inputted by GUI interface such as Pruning threshold, Network suppression threshold, etc.

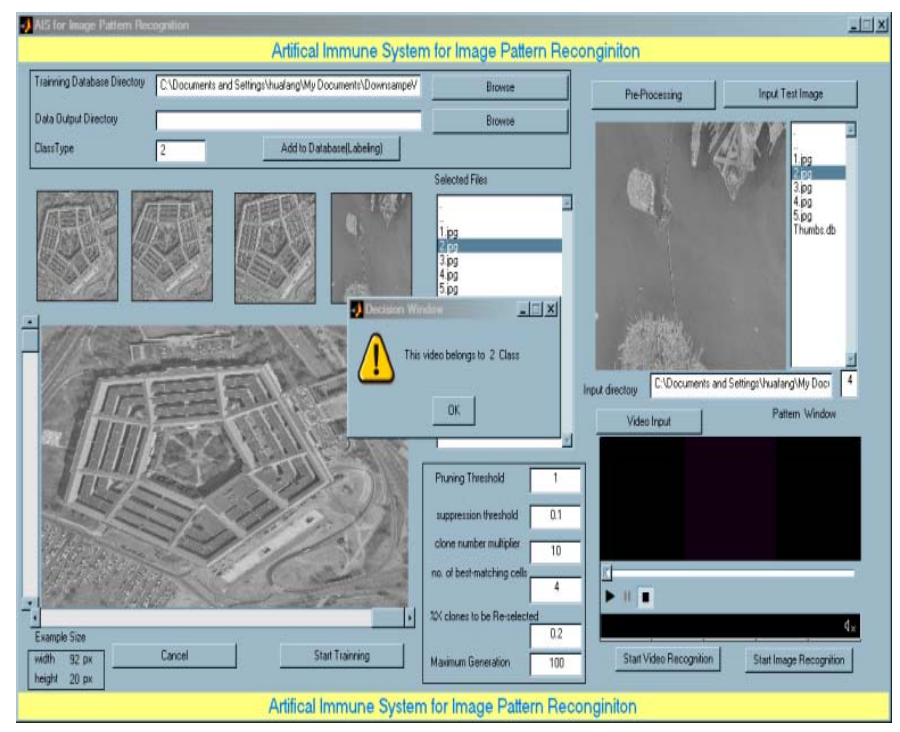

Figure 4. Immune Network-based GUI interface

Firstly, we conduct simulations of the IRIS data [18] in order to check the proposed designed immune network based on classifier's correctness and efficiency. In this simulation, only two points $(6,2.7,5.1,1.6)$ and $(6.3,2.5$, $4.9,1.5)$ are misclassified among 150 vectors, demonstrating that the proposed algorithm achieves as well as many other successful classification algorithms such as SVM and Decision trees.

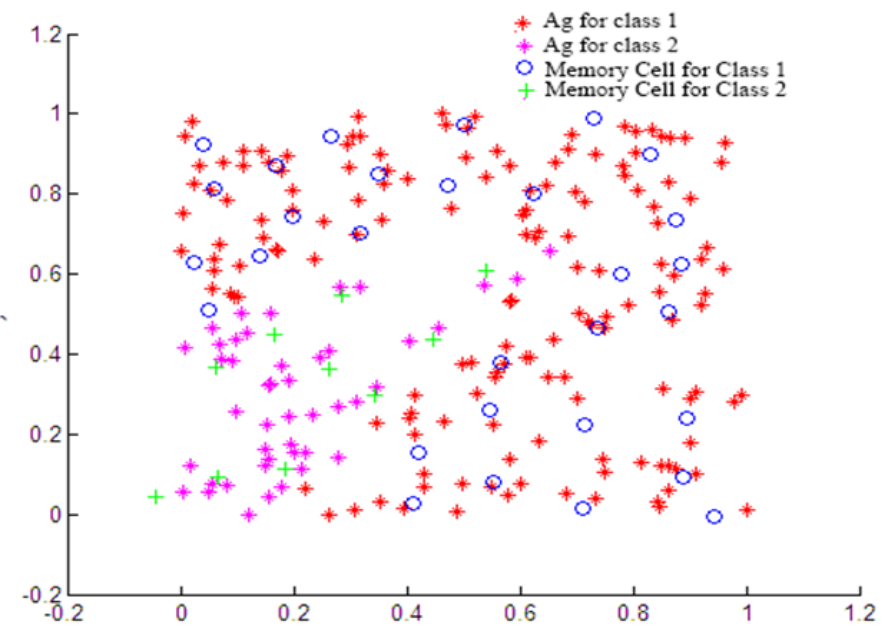

Figure 5. Artifical immune network data reduction representation for classification
The effectiveness of the data representation of designed immune network algorithm has also been validated in the experiment. Without losing generality, the experiment is based on the same data set as in [19]. As shown in Figure 5, fewer blue circle memory cells cover all the Ag points for Class 1, and fewer green "+" memory cells cover all the Ag for Class 2. What is also intriguing about Figure 5 is not just that there are far fewer memory cells than training items, but also that they are fairly easy to discern. Figure 6 demonstrates that the PCA reduction could efficiently reduce the data size while still keeping good recovered image quality. In Figure 6, the original large size image pixel data have been reduced to only 15 dimensions, each of which is being represented by a scalar.

$1024 \times 1024$ Orignal Image after Sampling Acceleration
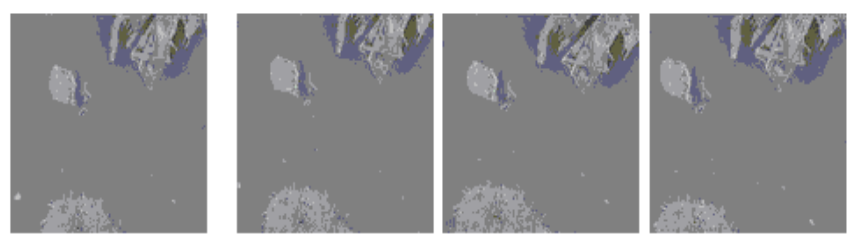

The Recovered Image after Dimensionality Reduction to 15 dimension
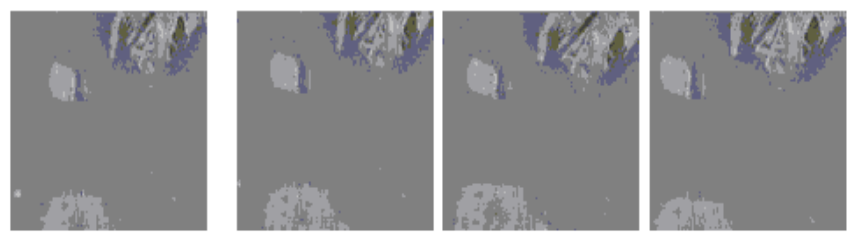

Figure 6. The recovered Land images after the dimensionality reduction to 15 dimensions by PCA

To evaluate the energy efficiency of the proposed scheme, we use the energy model described in [20] by B. Heinzelman as shown in the following:

$$
\begin{gathered}
E_{t}=L \cdot\left(E_{\text {elec }}+E_{f s} \cdot d^{k}\right) \\
E_{r}=L \cdot E_{\text {elec }}
\end{gathered}
$$

where $E_{t}$ and $E_{r}$ are the energy required by the sensor to transmit and receive a packet. $E_{\text {elec }}$ and $E_{f s}$ are the parameters of transmission/reception circuitry. Figure 7 shows the energy efficiency achievements. It has been demonstrated that the proposed PCA/AIS algorithms with sleep control mechanism achieve significantly more energy saving than non-sleep control schemes or other schemes without PCA reduction under the image pattern recognition requirements. With the further PCA dimensionality reduction and the further immune network memory cell representation, the energy efficiency has been achieved more due to the reduced size of image data load. With the further sampling acceleration, even more energy gain is achieved. We have also tested the proposed AIS- 
based with different types of video/image streaming. The result in Table 1 validates the proposed AIS algorithm classification efficiency for these different types of image/video data.

\section{CONCLUSIONS}

In this paper, we have proposed an energy efficient AISbased image recognition approach in WMSNs. Our study shows the great suitability of this method for large size image data processing and pattern recognition with minimum energy cost. The sleep control scheme driven with image recognition performance can achieve both recognition and energy efficiency. In the proposed approach, the PCA data reduction technique including the AIS memory cell representation has also improved the energy efficiency significantly. The AIS-based recognition is performed with high accuracy based on the HR image constructed through overlapping LR images, with practical sensor environments. The simulation results have proven the high degree of energy efficiency for the proposed approach with WMSN applications.

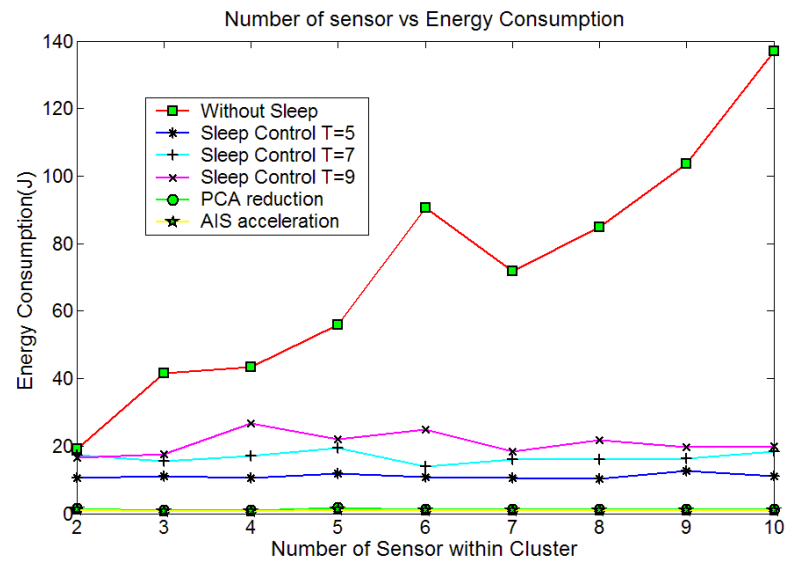

Figure 7. The Energy efficiency for the image recognition Vs Number of Sensor

Table 1. Image/Video Recognition Experiment

\begin{tabular}{|c|c|c|c|c|}
\hline $\begin{array}{c}\text { Test } \\
\text { video } \\
\text { type }\end{array}$ & Frame rate & $\begin{array}{c}\text { Number } \\
\text { training } \\
\text { sample }\end{array}$ & $\begin{array}{c}\text { Number of } \\
\text { Test video }\end{array}$ & $\begin{array}{c}\text { Correct } \\
\text { Classification }\end{array}$ \\
\hline Lake & $\begin{array}{c}3 \\
\text { frame/second }\end{array}$ & $\begin{array}{c}20 \\
\text { Pictures }\end{array}$ & 1 & Yes \\
\hline Land & $\begin{array}{c}3 \\
\text { frame/second }\end{array}$ & $\begin{array}{c}20 \\
\text { Pictures }\end{array}$ & 1 & Yes \\
\hline Car & $\begin{array}{c}10 \\
\text { frame/second }\end{array}$ & $\begin{array}{c}20 \\
\text { Pictures }\end{array}$ & 1 & Yes \\
\hline Airplane & $\begin{array}{c}5 \\
\text { frame/second }\end{array}$ & $\begin{array}{c}20 \\
\text { Pictures }\end{array}$ & 1 & Yes \\
\hline
\end{tabular}

\section{ACKNOWLEDGMENT}

This work was conducted at the Peter Kiewit Institute at the University of Nebraska-Lincoln partially supported by Raytheon Company.

\section{REFERENCES}

[1] R. C. Hardie, K. Barnard, and E. E. Armstrong, "Joint MAP registration and high-resolution image estimation using a sequence of under sampled images," IEEE Trans. Image Processing, Vol. 6, pp. 1621-1632, 1997.

[2] de Castro, L. N. \& Timmis, J. I., "Artificial Immune Systems: A Novel Paradigm to Pattern Recognition", in Artificial Neural Networks in Pattern Recognition, pp. 6784, 2002.

[3] I. T. Joliffe. Principal Component Analysis. SpringerVerlag, 1986.

[4] R. R. Schultz and R. L. Stevenson, "Extraction of highresolution frames from video sequences," IEEE Trans. Image Processing, Vol. 5, pp. 996-1011, 1996.

[5] L. N. de Castro and F. J. Von Zuben, "An evolutionary immune system network for data clustering," in Proc. 6th Brazilian Symp. Neural Networks, Brazil, 2000, pp. 84-89.

[6] L. N. de Castro and F. J. Von Zuben, "Learning and optimization using the clonal selection principal," IEEE Trans. Evol. Compu., Vol. 6, No. 3, pp. 239-251, 2002.

[7] Z. Zhu, S. Yang, G. Xu, X. Lin, D. Shi, "Fast Road Classification and Orientation Estimation Using OmniView Images and Neural Networks," IEEE Trans. Image Processing, Vol. 7, No. 8, pp. 1182-1197.

[8] A.H.S. Solberg, T.Taxt, A.K. Jain, “A Markov Random Field Model for Classification of Multisource Satellite Imagery," IEEE Trans. Geoscience and Remote Sensing, Vol. 34, No. 1, pp. 100-113.

[9] A. Chakrabarti, A.N. Rajagopalan, R. Chellappa, "Super-Resolution of Face Images Using Kernel PCABased Prior," IEEE Trans. On Multimedia, Vol. 9; No. 4, pp. 888-892, 2007.

[10] K. Kim, M. Franz, B. Schölkopf, "Iterative Kernel Principal Component Analysis for Image Modeling," IEEE Trans. Pattern Analysis and Machine Intelligence, Vol. 27, No. 9, pp. 1351-1366, 2005.

[11] S. Soro, W.B. Heinzelman, "On the coverage problem in video-based wireless sensor networks," in Proc. 2nd International Conference on Broadband Networks, 2005, Vol. 2, pp. 932- 939.

[12] A. Sinha, A. Chandrakasn, "Dynamic Power Management in Wireless Sensor Networks," IEEE Design \& Test of Computers, Vol. 18, No. 2, pp. 62-74, 2001.

[13] X. Yang, N. H. Vaidya, "A Wakeup Scheme for Sensor Networks: Achieving Balance between Energy Saving and End-to-end Delay," in Proc. 10th IEEE Real- 
Time and Embedded Technology and Applications Symposium (RTAS'04), pp. 19.

[14] J. Deng, Y. S. Han, W. B. Heinzelman, P. K. Varshney, "Scheduling Sleeping Nodes in High Density Cluster-based Sensor Networks," Mobile Networks and Applications, Vol. 10, No. 6, pp. 825-835, 2005.

[15] J-S. Wu, R-J. Chen, "An algorithm for computing the reliability of weighted-k-out-of-n systems", IEEE Trans. Reliability, Vol. 43, No.2, pp. 327-328, 1994.

[16]http://web.mit.edu/be.400/www/SVD/Singular_Value Decomposition.htm, Last Accessed June 07, 2008.

[17] L. N. de Castro, F. J. Von Zuben, "aiNet: An Artificial Immune Network for Data Analysis, " in Data Mining: A Heuristic Approach, H. A. Abbass, R. A. Sarker, and C. S. Newton (eds.), Idea Group Publishing, USA, Chapter XII, pp. 231-259.

[18] Iris data:

http://mlearn.ics.uci.edu/databases/iris/iris.data

[19] H.Wang, H.Fang, H.Sharif, Z.Wang "Nonlinear Classification by Genetic Algorithm with Signed Fuzzy Measure," in Proc. FUZZ-IEEE 2007, pp. 1-6.

[20] W. Heinzelman, A. Chandrakasan, H. Balakrishnan, "An Application-Specific Protocol Architecture for Wireless Microsensor Networks," IEEE Trans. Wireless Communications, Vol. 1, No. 4, pp. 660-670, 2002. 